\title{
Predicting the structure and vibrational frequencies of ethylene using harmonic and anharmonic approaches at the Kohn-Sham complete basis set limit
}

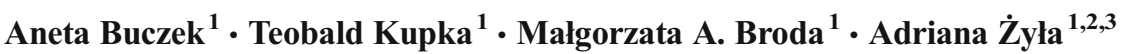

Received: 24 November 2015 / Accepted: 22 December 2015/Published online: 22 January 2016

(C) The Author(s) 2016. This article is published with open access at Springerlink.com

\begin{abstract}
In this work, regular convergence patterns of the structural, harmonic, and VPT2-calculated anharmonic vibrational parameters of ethylene towards the Kohn-Sham complete basis set (KS CBS) limit are demonstrated for the first time. The performance of the VPT2 scheme implemented using density functional theory (DFT-BLYP and DFT-B3LYP) in combination with two Pople basis sets $\left(6-311++\mathrm{G}^{* *}\right.$ and $\left.6-311++\mathrm{G}(3 \mathrm{df}, 2 \mathrm{pd})\right)$, the polarizationconsistent basis sets pc- $n$, aug-pc- $n$, and pcseg- $n(n=0,1,2$, $3,4)$, and the correlation-consistent basis sets cc-pVXZ and aug-cc-pVXZ $(X=\mathrm{D}, \mathrm{T}, \mathrm{Q}, 5,6)$ was tested.

The BLYP-calculated harmonic frequencies were found to be markedly closer than the B3LYP-calculated harmonic frequencies to the experimentally derived values, while the calculated anharmonic frequencies consistently underestimated the observed wavenumbers. The different basis set families gave very similar estimated values for the CBS parameters. The anharmonic frequencies calculated with B3LYP/aug-pc-3 were consistently significantly higher than those obtained
\end{abstract}

Electronic supplementary material The online version of this article (doi:10.1007/s00894-015-2902-z) contains supplementary material, which is available to authorized users.

Aneta Buczek

abuczek@uni.opole.pl

Teobald Kupka teobaldk@gmail.com

1 Faculty of Chemistry, University of Opole, 48, Oleska Street, 45-052 Opole, Poland

2 Department of Biosystematics, University of Opole, Oleska 22, 45-052 Opole, Poland

3 Present address: Institute of Physics, Adam Mickiewicz University in Poznan, 65, Umultowska Street, 61-614 Poznań, Poland with the pc-3 basis set; applying the aug-pcseg- $n$ basis set family alleviated this problem. Utilization of B3LYP/augpcseg- $n$ basis sets instead of B3LYP/aug-cc-pVXZ, which is computationally less expensive, is suggested for mediumsized molecules. Harmonic BLYP/pc-2 calculations produced fairly accurate ethylene frequencies.

Keywords Ethylene - Anharmonic vibration - VPT2 - DFT CBS · Polarization-consistent basis sets .

Correlation-consistent basis sets

\section{Introduction}

Theoretical modeling of structures and vibrational frequencies has often been performed in combination with analysis of experimental IR and Raman spectra. Ethylene is the smallest compound with a carbon-carbon double bond, and can be considered a simplified model of polyenes [1,2]. The local environments of the $\mathrm{C}=\mathrm{C}$ bonds present in numerous natural and synthetic compounds, including polyenes in red corals, have routinely been studied using vibrational spectroscopy [2].

Vibrational frequencies calculated using SCF, DFT, and MP2 are overestimated because the effects of anharmonicity are omitted [3-5]. For example, SCF predicts $\mathrm{C}-\mathrm{H}, \mathrm{N}-\mathrm{H}$, and $\mathrm{O}-\mathrm{H}$ stretching vibrations that are about $5-10 \%$ higher than those actually observed. This deficiency in SCF, DFT, and MP2 has been pragmatically corrected by introducing frequency scaling [4, 6, 7]. Unfortunately, the method of calculation and the basis set are important considerations during the tedious estimation of proper, empirically derived scaling factors.

Instead of using scaling factors, vibrational analysis based on the inclusion of an anharmonic correction is better justified 
theoretically. Among several anharmonic approaches (including VSCF [8-11], VCI [12-14], and VCC [15]), the recent implementation of VPT2 [16-20] has proven to be very robust and efficient when investigating small and medium-sized molecules. Obviously, such an anharmonic approach is significantly more computationally expensive than a simple harmonic model. For this reason, anharmonic calculations are mainly performed using DFT [21, 22] and relatively small basis sets, such as Pople basis sets or early members of the series of Dunning's correlation-consistent basis sets [23-26].

A family of correlation-consistent basis sets, cc-pVXZ, and another similar family with additional augmented diffuse functions (aug-cc-pVXZ), have been proposed by Dunning [23-26]. The main feature of these basis sets is the regular and smooth convergence of atomic and molecular energies towards the complete basis set (CBS) limit. Moreover, it has been shown that the CBS values for energy and some other parameters that are directly related to the energy can be estimated using two- and three-parameter formulae. The best agreement between these theoretically calculated values and the corresponding experimentally derived values was obtained when the results calculated using the most complete basis sets $(X>2)$ were fitted. Two additional basis set series that are similar to those of Dunning are available: the polarizationconsistent [27-32] and XZP [33] basis sets. The same limiting parameter values (CBS) were reportedly [34-36] obtained from noncorrelated and correlated calculations performed using Dunning's and Jensen's basis sets. It is important to note that the results converge more quickly when polarizationconsistent basis sets are applied.

Correlation-consistent basis sets have been used to accurately calculate the geometries and vibrational parameters of several small molecules at the coupled cluster level of theory $[37,38]$. Approaches based on correlation-consistent basis sets and coupled cluster calculations are very expensive computationally and are thus unsuitable for larger molecular systems. Unfortunately, the systematic calculation of molecular systems using polarization-consistent basis sets is not yet commonplace. Besides, the combination of anharmonic models for frequency calculations with polarizationconsistent basis sets is still the source of debate.

Another issue that needs to be explored is the convergence pattern toward the CBS limit of Kohn-Sham wavelengths calculated using the pc- $n$ and cc-pVXZ basis sets. In our recent studies [39-42], we demonstrated that $\mathrm{H}_{2} \mathrm{O}, \mathrm{CH}_{2} \mathrm{O}$, and $\mathrm{H}_{2} \mathrm{NCHO}$ harmonic and anharmonic frequencies converged regularly toward the $\mathrm{KS}$ limit when Jensen-type basis sets were used. These basis sets were originally designed as general-purpose basis sets to be used in particular to predict Hartree-Fock and Kohn-Sham energies and structural and vibrational parameters. In subsequent studies [42], we noticed that anharmonic frequencies showed irregular behavior in polar solvents when the PCM solvation model was used [43]. In addition, we observed [41] a simple relation between harmonic and anharmonic $\mathrm{C}=\mathrm{O}$ stretching frequencies (the corresponding wavenumbers differ by about $31 \mathrm{~cm}^{-1}$ ).

Although a number of studies on the structure and harmonic properties of ethylene have been reported [38, 44-47], there has been no systematic use of polarization-consistent basis sets, particularly the newly designed segmented contracted ones, at the DFT level of theory. Thus, due to the importance of ethylene as the basic building block of linear polyenes, we thought that it would be interesting to find an efficient way of accurately calculating its structural and vibrational properties. Therefore, in the study reported in the present paper, we compared the accuracy of the harmonic and anharmonic frequencies of ethylene in the gas phase calculated using recently developed families of Jensen basis sets with the results obtained using traditional Dunning basis sets. We tested the performance of the harmonic model, and the computationally expensive anharmonic approach, using a well-performing hybrid density functional (B3LYP). The performance of B3LYP in predicting ethylene frequencies was compared to the results obtained with the BLYP functional [48], benchmark literature values, and reported experimental data.

\section{Computational methods}

All DFT calculations using the popular B3LYP [49-51] and BLYP [50-52] density functionals in combination with 6-311++G** and 6-311++G(3df,2pd) Pople-type basis sets $[53,54]$, Jensen's polarization-consistent pc- $n$, aug-pc- $n$ [28, 29], pcseg- $n$, and aug-pcseg- $n$ [55] (for $n=0,1,2,3,4$ ) basis sets, and Dunning's correlation-consistent cc-pVXZ and augcc-pVXZ [23-26] basis sets were performed with the D.01 version of the Gaussian 09 program [56]. The Jensen basis sets were downloaded from EMSL [57-59]. It is important to note that all of the B3LYP-calculated and BLYP-calculated structural parameters and harmonic frequencies of ethylene obtained using the A.02 [60] version of Gaussian 09 were practically identical to those obtained using the D.01 [56] version. However, the anharmonic frequencies for ethylene obtained with the latter version were somewhat smaller [48], resulting in better agreement of the B3LYP(anharmonic)predicted $\mathrm{C}=\mathrm{C}$ stretching frequency with the corresponding experimental value.

Unconstrained geometry optimization of ethylene was performed using very tight convergence criteria and a very large grid, as specified using the keyword INT(GRID=150590). Harmonic and anharmonic frequencies were then calculated. The anharmonic wavenumbers were obtained via Barone's implementation of vibrational second-order perturbation theory (VPT2) [18] in the latest (D.01 [56]) version of Gaussian 09. Detailed assignment of ethylene vibrational modes was performed based on the results of previous studies [61-63] 
(see also Table S1 in the "Electronic supplementary material," ESM).

Moreover, to assess the performance of the chosen density functional in predicting structural and vibrational parameters of ethylene, calculations using the pc- $n$, aug-pc- $n$, cc-pVXZ, and aug-cc-pVXZ basis sets were performed, and the individual results, $Y(x)$, were extrapolated toward the Kohn-Sham (KS) complete basis set limit $Y(\infty)$ using the three-parameter $[64,65]$ and two-parameter $[66,67]$ formulae

$Y(x)=Y(\infty)+A \exp (-x / B)$

$Y(x)=Y(\infty)+A / x^{3}$

The extrapolated value $Y(\infty)$ is the optimal estimate of the predicted structural or vibrational parameter [34, 39, 40, 67, 68] for a very large (e.g., infinite) zeta or cardinal number $x$, and $A$ and $B$ are fitting parameters. In addition, to check the convergence of the anharmonic $v(\mathrm{C}=\mathrm{C})$ mode for ethylene, the calculations were repeated using the newly introduced segmented contracted polarization-consistent basis sets pcseg- $n$ and aug-pcseg- $n$ [55]. A similar strategy has been used in previous studies $[34,39,69,70]$ to accurately predict the structural, vibrational, and NMR parameters of small molecules at the HF, DFT, MP2, and CCSD(T) levels of theory at the complete basis set limit (CBS).

Please note that, for the sake of brevity, the basis sets cc-pVXZ, aug-cc-pVXZ, pc- $n$, aug-pc- $n$, pcseg- $n$, and aug-pcseg- $n$ are sometimes abbreviated to "XZ," "aXZ," "pcn," "apcn," "pcsegn," and "apcsegn," respectively, in the figures for this paper.

\section{Results and discussion}

\section{Structure of ethylene}

The structure of ethylene was fully optimized using the B3LYB and BLYP density functionals in combination with the two fairly complete and flexible Pople-type basis sets $6-311++\mathrm{G}^{* *}$ and $6-311++\mathrm{G}(3 \mathrm{df}, 2 \mathrm{pd})$, a selected series of Jensen's polarization-consistent basis sets, and Dunning's correlation-consistent basis sets. Next, using Eq. 1 or 2, the CBS values of the structural parameters of ethylene were estimated from the last two (or three) points obtained for the corresponding largest Jensen and Dunning basis sets. The resulting structural parameter values are compared in Table 1 with the best corresponding values given in the literature [44-46], including those for the semiempirical structure [47] of $\mathrm{C}_{2} \mathrm{H}_{4}$.

Figure 1 shows the $\mathrm{C}-\mathrm{C}$ and $\mathrm{C}-\mathrm{H}$ bond lengths and $\mathrm{C}-\mathrm{C}-$ $\mathrm{H}$ angle of ethylene obtained with the $6-311++\mathrm{G}^{* *}, 6-311++$ $\mathrm{G}(3 \mathrm{df}, 2 \mathrm{pd})$, pc- $n$, and aug-pc- $n$ basis sets. The best fits, as well as the estimated CBS values, obtained using Eq. 2 with the three largest basis sets are also shown. The $\mathrm{C}-\mathrm{C}$ bond length was found to be insensitive to the basis set size, while the $\mathrm{C}-\mathrm{H}$ bond and $\mathrm{C}-\mathrm{C}-\mathrm{H}$ angle did not fully converge when pc-2 and aug-pc-2 were used. All of the calculated structural parameters are gathered in Table S2A, B in the ESM.

It is clear from Fig. 1 that the structural parameters of ethylene calculated using the BLYP and B3LYP density functionals almost converged when the pc-2 and aug-pc-2 basis sets were applied, but the results were far from the estimated CBS values (i.e., accidentally over- or underestimated) when smaller basis sets were used. Augmenting the basis set with diffuse functions did not improve the results (e.g., compare the pc-3 data with the aug-pc-3 data, or the pc-4 data with the aug-pc-4 data).

As expected, at the complete basis set limit, the values of all of the structural parameters depended only on the density functional used (the pc- $n$, aug-pc- $n$, pcseg- $n$, and aug-pcseg- $n$ series of basis sets all gave very similar results; see Table 1 and Table S2A, B in the ESM for the results obtained for all of the basis sets considered in this work). For example, the differences between estimates given by B3LYP and BLYP at the Kohn-Sham limit in combination with different families of basis sets for the lengths of the $\mathrm{C}-\mathrm{C}$ and $\mathrm{C}-\mathrm{H}$ bonds in ethylene are $0.0002,0.0003$, and $0.0003,0.0005 \AA$, while the estimates afforded by these two functionals for the $\mathrm{C}-\mathrm{C}-\mathrm{H}$ angle differ by only $0.01^{\circ}$.

The B3LYP functional underestimates the $\mathrm{C}=\mathrm{C}$ bond length (given that the reference semiempirical value [47] is $1.3305 \AA$ ) by $0.007 \AA$, while BLYP overestimates the reference value by $0.003 \AA$. The corresponding deviations obtained when the smallest basis set is used $\left(6-311++\mathrm{G}^{* *}\right)$ are -0.002 and $0.008 \AA$, respectively. For the $\mathrm{C}-\mathrm{H}$ bond length, the deviations of the B3LYP- and BLYP-calculated values from the reference value are 0.001 and $0.007 \AA$. Somewhat larger deviations are seen when the smallest basis set $\left(6-311++\mathrm{G}^{* *}\right)$ is applied: 0.005 and $0.011 \AA$. Interestingly, the hybrid B3LYP and pure BLYP density functionals yield very similar deviations regardless of the basis set used: they overestimate the $\mathrm{C}-\mathrm{C}-\mathrm{H}$ angle by $0.29^{\circ}$ and $0.34^{\circ}$, respectively. Therefore, we can conclude that the results for the structural parameters of ethylene gathered in Table 1 indicate that B3LYP is somewhat better than BLYP at predicting the geometry of ethylene. It is important to note that the values of the structural parameters of ethylene almost converged when the pc-2 and aug-pc-2 basis sets were used. The $6-311++\mathrm{G}^{* *}$ basis set, which can feasibly be applied to large molecules such as polyenes [1, 71], is somewhat less accurate than (computationally very demanding) CBS calculations.

\section{Convergence patterns of harmonic and anharmonic vibrations of ethylene}

Due to differences in the anharmonicities of the normal modes of ethylene, high- and low-frequency harmonic vibrations are 
Table 1 Structural parameters of ethylene, calculated using the BLYP and B3LYP density functionals in combination with selected basis sets

\begin{tabular}{llll}
\hline Method/basis set & $\mathrm{C}=\mathrm{C}$ length $(\AA)$ & $\mathrm{C}-\mathrm{H}$ length $(\AA)$ & $\mathrm{C}-\mathrm{C}-\mathrm{H}$ angle $\left(^{\circ}\right)$ \\
\hline B3LYP & & & \\
6-311++G** & 1.3289 & 1.0850 & 121.74 \\
6-311++G(3df,2pd) & 1.3247 & 1.0823 & 121.74 \\
CBS (pc- $n$ ) & 1.3240 & 1.0815 & 121.73 \\
CBS (aug-pc-n) & 1.3239 & 1.0815 & 121.74 \\
CBS (pcseg- $n$ ) & 1.3241 & 1.0818 & 121.73 \\
CBS (aug-pcseg- $n$ ) & 1.3241 & 1.0818 & 121.74 \\
CBS (cc-pVXZ) & 1.3241 & 1.0818 & 121.74 \\
CBS (aug-cc-pVXZ) & 1.3241 & 1.0818 & 121.74 \\
BLYP & & & 121.79 \\
6-311++G** & 1.3384 & 1.0915 & 121.79 \\
6-311++G(3df,2pd) & 1.3341 & 1.0887 & 121.78 \\
CBS (pc- $n$ ) & 1.3335 & 1.0877 & 121.79 \\
CBS (aug-pc-n) & 1.3334 & 1.0877 & 121.79 \\
CBS (cc-pVXZ) & 1.3337 & 1.0882 & 121.79 \\
CBS (aug-cc-pVXZ) & 1.3337 & 1.0882 & 121.44 \\
From the literature: & & & $\mathbf{1 2 1 . 4 5 ( 1 0 )}$ \\
Best composite theory ${ }^{\mathrm{a}}$ & 1.3307 & 1.0809 & \\
Best composite theory & 1.3308 & 1.0803 & $\mathbf{1 . 0 8 0 5 ( \mathbf { 1 0 } )}$ \\
Semi-empirical ${ }^{\mathbf{c}}$ & $\mathbf{1 . 3 3 0 5 ( \mathbf { 1 0 } )}$ & & \\
\hline
\end{tabular}

The CBS values were estimated using a two-parameter fit (Eq. 2)

${ }^{\mathrm{a}}$ From [44]; ${ }^{\mathrm{b}}$ from [46]; ${ }^{\mathrm{c}}$ from [47]; calculated with different levels of accuracy [45]. To permit a comparison of theoretically predicted frequencies with the corresponding observed frequencies, Table S1 in the ESM shows experimental (fundamental) frequencies for ethylene, their symmetries, and their assignments [62, 63, 72]. Among the 12 vibrations shown, the four high-frequency ones (due to $\mathrm{C}-\mathrm{H}$ stretching) are overestimated to the greatest degree in comparison to the experimental values $[62,72]$. The characteristic $\mathrm{C}=\mathrm{C}$ stretch vibration is overestimated to a lesser degree, and the remaining modes (below $1500 \mathrm{~cm}^{-1}$ ) are expected to be predicted more accurately by theory.

To show the nonuniform accuracy of the vibrational frequency values predicted using the density functionals and basis sets studied in this work for both the harmonic and anharmonic (VPT2) models, we now consider individual vibrational frequency deviations and RMS deviations. For brevity, all of the frequency values are listed in Tables S3A-S8A in the ESM. Moreover, all of the calculated vibrational frequencies were plotted as a function of basis set size, and the corresponding CBS values were estimated using the two largest basis sets (these are not shown here for the sake of brevity).

Since $\mathrm{C}=\mathrm{C}$ stretching is the most important diagnostic mode in the vibrational (Raman) spectra of polyenes, we analyzed the performance of the selected theoretical models used to predict this mode. Figure 2a shows the influence of the basis set on the magnitude of $\mathrm{C}=\mathrm{C}$ stretching; results were calculated using the B3LYP and BLYP density functionals in combination with the pc- $n$ and aug-pc- $n$ basis sets. In this case, the pc- $n$ and aug-pc- $n$ basis sets perform equally well for $n \geq 2$. Results showing a considerable amount of scatter are obtained for $n=0$ and 1, as well as for the two selected Pople-type basis sets. Table 2 presents the deviations in the harmonic and anharmonic $\mathrm{CBS}$ values of the $\mathrm{C}=\mathrm{C}$ stretching vibrational frequency from the corresponding experimentally derived value (see also Tables $\mathrm{S} 1$ and S8B in the ESM). Note that the $\mathrm{CBS}$ values for the $\mathrm{C}=\mathrm{C}$ stretching vibrational frequency are overestimated (by about $64 \mathrm{~cm}^{-1}$ ) by a simple harmonic model when using the B3LYP density functional (Fig. 2a and Table 2). On the other hand, employing the BLYP density functional with the pc- $n$, aug-pc- $n$, cc-pVXZ, and augcc-pVXZ basis sets leads to fairly accurate predictions for the harmonic $\mathrm{C}=\mathrm{C}$ stretching vibrational frequency (the experimental value of $1625.4 \mathrm{~cm}^{-1}$ is overestimated by less than $10 \mathrm{~cm}^{-1}$ ).

By contrast, the BLYP-calculated anharmonic frequencies obtained using the pc- $n$ and aug-pc- $n$ basis sets (see Fig. 2b) are significant underestimates (about $33 \mathrm{~cm}^{-1}$ too low) compared to the experimentally derived value $[62,72]$. On the other hand, the corresponding anharmonic B3LYP/CBS values obtained with the pc- $n$ and aug-pc- $n$ basis sets overestimate the actual (experimental) value by only about 20 and $7 \mathrm{~cm}^{-1}$, respectively. However, closer inspection of the latter 
a

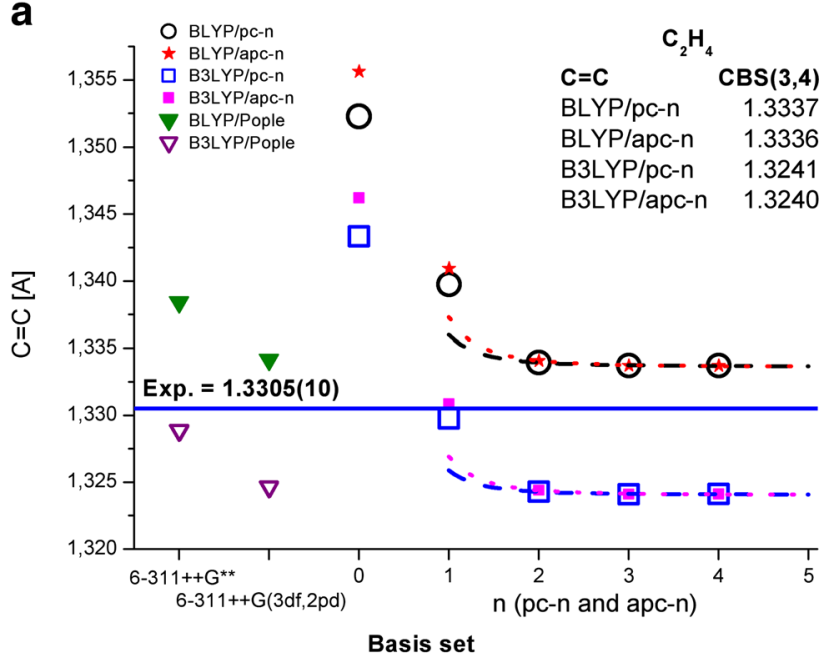

b

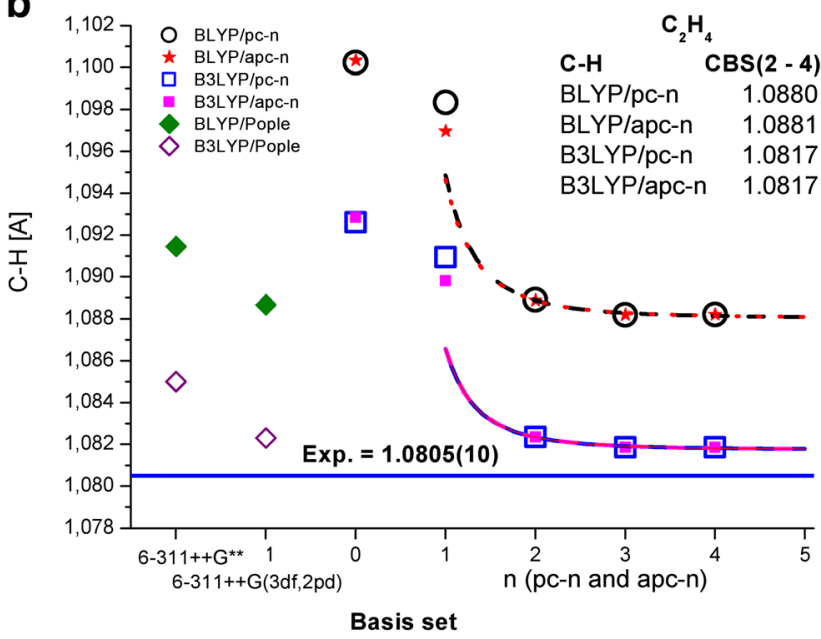

C

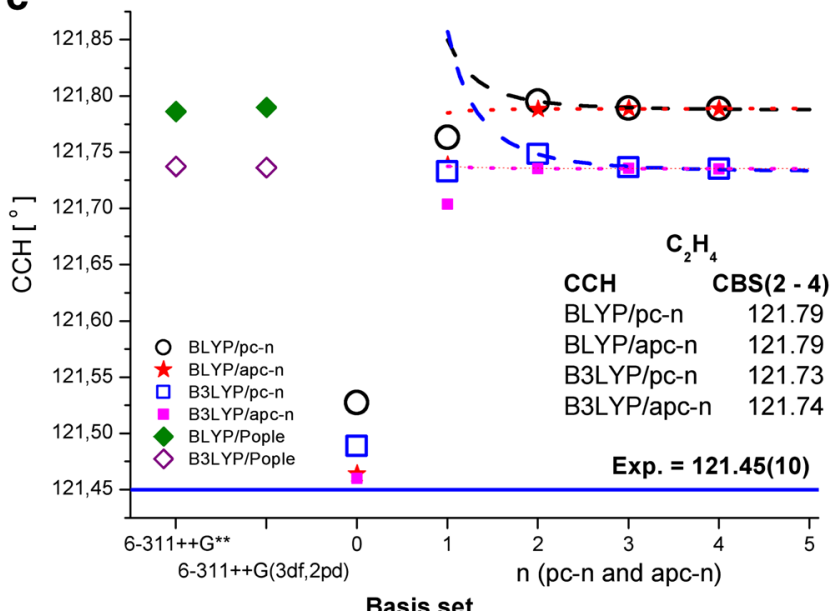

Fig. 1a-c Sensitivity of the BLYP- and B3LYP-calculated ethylene $\mathrm{C}=\mathrm{C}$ (a) and $\mathrm{C}-\mathrm{H}$ (b) bond lengths, as well as the $\mathrm{C}-\mathrm{C}-\mathrm{H}$ bond angle (c), to the quality of the basis set. Continuous lines mark experimental values [47], and the results of CBS fits are also shown

results suggests that the $\mathrm{B} 3 \mathrm{LYP} /$ aug-pc-3 result is about $20 \mathrm{~cm}^{-1}$ higher than the pc-3 result. Indeed, all 12 anharmonic a

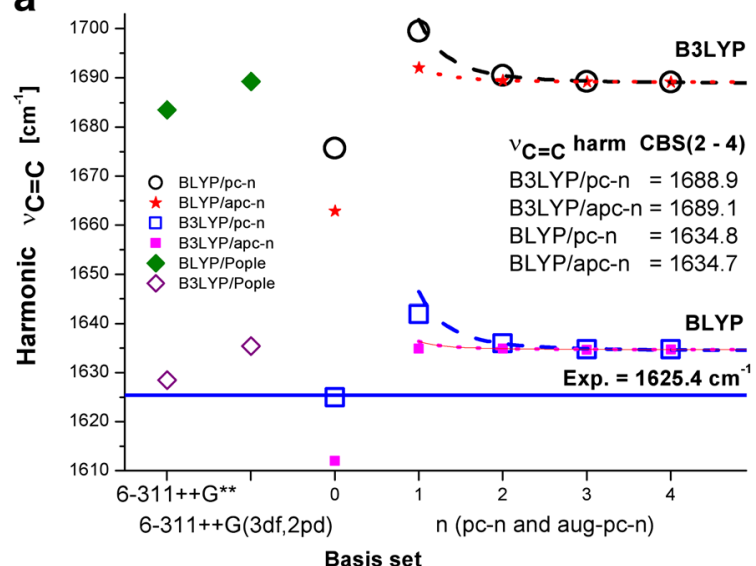

b

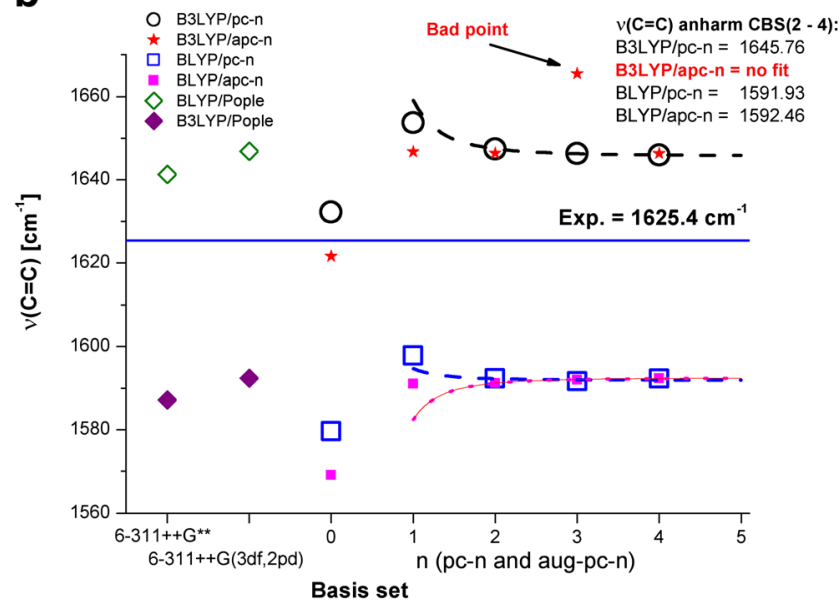

Fig. 2a-b Sensitivity of the BLYP-and B3LYP-calculated values for the ethylene $v(C=C)$ vibrational frequency to the quality of the basis set when predictions are made using the harmonic (a) and anharmonic (b) approximations. A continuous line indicates the experimental value [47], and the CBS fitting results are also shown

frequencies of ethylene obtained at the B3LYP/aug-pc-3 level of theory are significantly overestimated in comparison with those obtained with the pc-3 basis set. Thus, two-point fitting cannot be performed in this case (this entry is marked in red in

Table 2 Deviations of calculated CBS values for the ethylene $\mathrm{C}=\mathrm{C}$ stretching vibrational frequency from the corresponding experimentally derived value $[62,63,72]$

\begin{tabular}{llllll}
\hline Basis set & \multicolumn{3}{l}{ BLYP } & & \multicolumn{2}{l}{ B3LYP } \\
\cline { 2 - 3 } \cline { 5 - 6 } \cline { 5 - 6 } & Harmonic & Anharmonic & & Harmonic & Anharmonic \\
\hline pc- $n$ & 9.36 & -32.67 & & 63.65 & 20.25 \\
aug-pc- $n$ & 9.41 & -32.80 & & 63.70 & 7.00 \\
cc-pVXZ & 8.74 & -37.26 & & 63.74 & 23.27 \\
aug-cc-pVXZ & 9.14 & -37.75 & & 63.69 & 9.22 \\
pcseg- $n$ & - & - & 63.58 & 18.31 \\
aug-pcseg- $n$ & - & - & 63.63 & 16.94 \\
\hline
\end{tabular}


Fig. 2b). On the other hand, it is important to stress that the anharmonic frequencies calculated at the BLYP/aug-pc- $n$ level of theory show the correct behavior (regular convergence).

This poor performance of B3LYP/aug-pc-3 when it was used to calculate anharmonic frequencies (see also Table S5B in the ESM) prompted us to check the performance of the newly released (and refined) pcseg- $n$ and aug-pcseg- $n$ basis sets [55], as well as Dunning's cc-pVXZ and aug-cc-pVXZ series of basis sets [23-26]. The relevant results are gathered in Table S6A, B in the ESM (the ESM presents frequencies calculated with both of the density functionals considered in this work and all of the selected basis sets; see Tables S3A-S8A). None of the other basis sets exhibited the poor performance shown by B3LYP/aug-pc-3 when calculating anharmonic frequencies.

We are aware that the good performance of BLYP in predicting the ethylene $v(\mathrm{C}=\mathrm{C})$ harmonic frequency could be an effect of fortuitous error cancelation [48]. Nevertheless, this density functional may represent a pragmatic choice for investigating a set of compounds containing $\mathrm{C}=\mathrm{C}$ bonds. Thus, we consider the BLYP density functional to be suitable for fairly accurate modeling of the structures and harmonic vibrational parameters of larger polyenes. The abovementioned hypothesis is also supported by the results of our recent studies on difluorinated ethylene isomers [48] and perfluorinated compounds [73], which are used as precursors for plastic optical fibers (POF).

It is important to note that the accuracies of the methods and basis sets considered in Table 2 when they were used to predict the $\mathrm{C}=\mathrm{C}$ stretching vibrational frequency are also valid for all other individual vibrations. Indeed, as shown in Table 3, when all vibrations are considered, the calculated RMS deviation is about $65 \mathrm{~cm}^{-1}$ when the BLYP anharmonic model is used, but only about $26 \mathrm{~cm}^{-1}$ when the BLYP harmonic model is employed. The reverse situation is observed for the B3LYP calculated harmonic and anharmonic frequencies (RMS devations of about 77 and $20 \mathrm{~cm}^{-1}$ ).

Table 3 RMS deviations of calculated CBS frequencies for ethylene from the corresponding experimentally derived frequencies [62, 63, 72]

\begin{tabular}{llllll}
\hline Basis set & \multicolumn{3}{l}{ BLYP } & & \multicolumn{2}{l}{ B3LYP } \\
\cline { 2 - 3 } \cline { 5 - 6 } \cline { 5 - 6 } & Harmonic & Anharmonic & & Harmonic & Anharmonic \\
\hline pc- $n$ & 26.04 & 62.96 & & 76.90 & 16.42 \\
aug-pc- $n$ & 26.04 & 63.53 & & 76.92 & $58.25^{\mathrm{a}}$ \\
cc-pVXZ & 25.99 & 62.56 & & 76.90 & 18.15 \\
aug-cc-pVXZ & 26.13 & 67.15 & & 76.96 & $\sim 31.5^{\mathrm{a}}$ \\
pcseg- $n$ & - & - & & 76.86 & 20.87 \\
aug-pcseg- $n$ & - & - & 76.83 & 23.63 \\
\hline
\end{tabular}

${ }^{\text {a }}$ The RMS deviations are high in these cases because the values obtained using aug-pc-3 are much too high or because there is considerable scatter in the results afforded by aug-cc-pVXZ
The current work considered only one molecular system (ethylene) and two density functionals (B3LYP and BLYP). Such a limited study cannot warrant extrapolating the results obtained here to other molecules. However, in the future it would be interesting to test our approach on a set of polyenes containing conjugated sets of double and single carbon-carbon bonds.

\section{Convergence patterns of the total and ZPV energies of ethylene}

The molecular energy and the zero-point vibrational energy are two of the most commonly encountered parameters in theoretical thermochemistry [5, 23, 26, 67, 74]. Obviously, since we have considered different calculation methods (for example, density functionals) in this work, we are not interested in absolute energy values, only energy differences. The correlation-consistent basis sets developed by Dunning and coworkers were designed to show the regular convergence
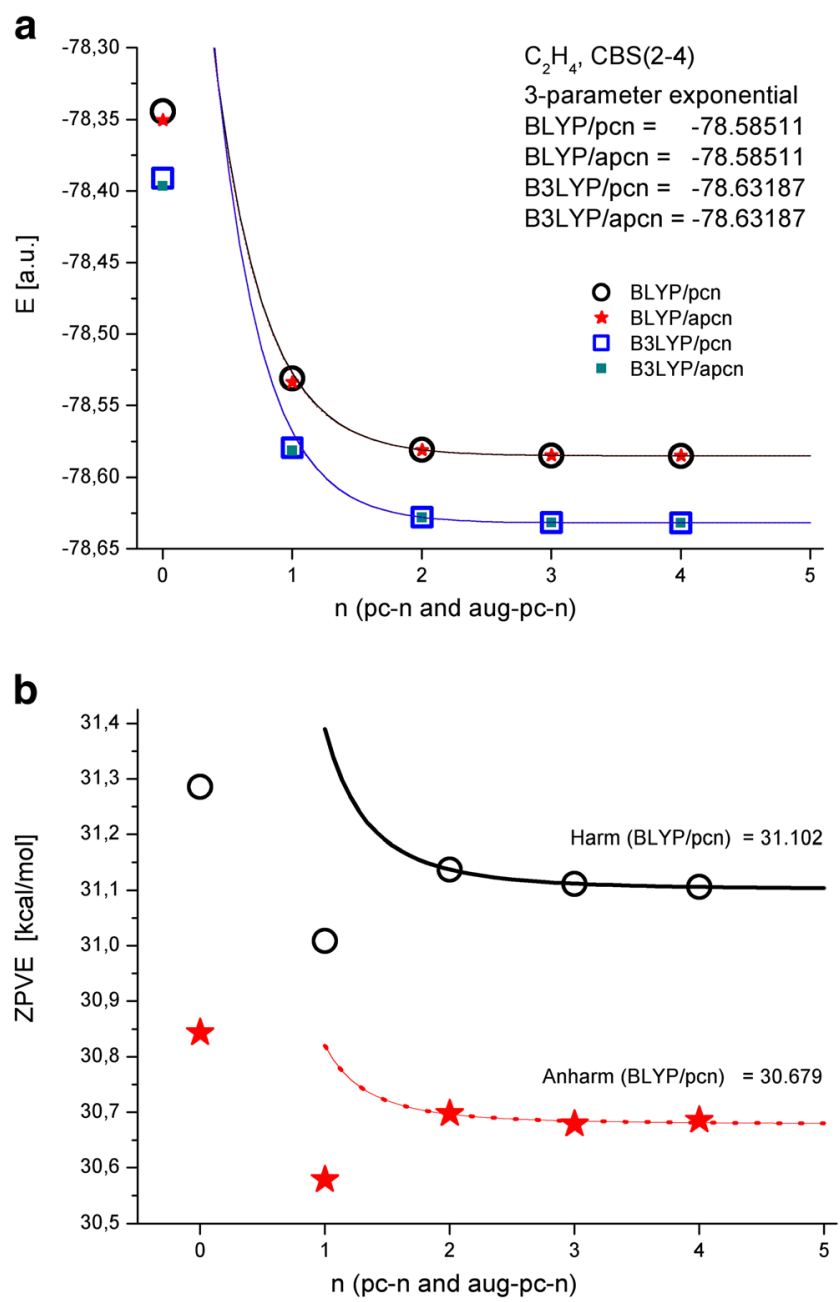

Fig. 3 a Convergence of the BLYP- and B3LYP-calculated energies of ethylene toward the Kohn-Sham limit when using the pc- $n$ and aug-pc- $n$ basis set families. b Convergence of the raw and anharmonic ZPVE values calculated using BLYP/pc- $n$ 
Table 4 Calculated CBS values of the raw and anharmonic ZPV energies $(\mathrm{kcal} / \mathrm{mol})$

\begin{tabular}{llllll}
\hline \multirow{2}{*}{ Basis set } & \multicolumn{2}{l}{ BLYP } & \multicolumn{3}{l}{ B3LYP } \\
\cline { 2 - 3 } \cline { 5 - 6 } \cline { 5 - 6 } & Harmonic & Anharmonic & & Harmonic & Anharmonic \\
\hline cc-pVXZ & 31.112 & 30.678 & 31.957 & 31.567 \\
aug-cc-pVXZ & 31.109 & 30.587 & & 31.567 & $\sim 31.5^{\mathrm{a}}$ \\
pc- $n$ & 31.361 & 30.941 & & 32.208 & 31.776 \\
aug-pc- $n$ & 31.368 & 30.957 & & 32.216 & $31.796^{\mathrm{a}}$ \\
pcseg- $n$ & - & - & 31.937 & 31.518 \\
aug-pcseg- $n$ & - & - & 31.944 & 31.528 \\
From the literature: & & & & & \\
CCSD(T)/aQZ & $32.048^{\mathrm{b}}$ & & & & \\
\hline
\end{tabular}

${ }^{\mathrm{a}}$ There was scatter in some of the B3LYP/aug-cc-pVXZ results, and the aug-pc-4 result is shown for aug-pc- $n$ because the aug-pc-3 result is significantly larger than the pc-3 result

b The CCSD(T)/aQZ-calculated harmonic result from NIST is shown for comparison [76]

of molecular energy and parameters directly related to energy toward the complete basis set limit in correlated calculations [23-26, 67, 68]. Among the CBS-estimated parameters are structural, magnetic, and vibrational parameters. Since these parameters are calculated as derivatives (or second derivatives) of the energy, the convergence curves are not very smooth, so the results for the initial (smallest) basis sets are often not used in the CBS fitting procedure $[26,34,70,75]$. Thus, we expected the convergence of the ZPV energy of ethylene to be significantly worse than the convergence of its total energy. Figure $3 \mathrm{a}$ shows the influence of the basis set on the B3LYPand BLYP-calculated energies of ethylene. In this case, a three-parameter exponential fit based on the results obtained using the three largest basis sets $(n=2,3,4)$ was employed. Note that the least accurate result, obtained using the $n=0$ basis set, is clearly far from the fit line. Also note that the CBS energies calculated with the pc- $n$ and aug-pc- $n$ basis sets were identical.

The corresponding BLYP-calculated raw and anharmonic ZPV energies show similar patterns starting from $n=2$ (Fig. 3b). The three-parameter fit lines based on the results obtained using the three largest basis sets are also plotted. The CBS values of the ZPV energy obtained using a simply harmonic model are overestimated (by about $1.3 \%$ ). A similar relation between the raw and anharmonic ZPV values is observed when the B3LYP density functional is employed (not shown). Table 4 lists the estimated CBS values of the raw and anharmonic ZPV energies, calculated using the BLYP and B3LYP density functionals and the selected basis set families. The DFT values are compared with benchmark CCSD(T)/aug-cc-pVQZ results reported in the literature [76]. As also seen for the absolute energy of ethylene, the ZPV values calculated with B3LYP and BLYP differ. The raw ZPV energies calculated with the B3LYP density functional and different basis sets are larger than the corresponding anharmonic values. The same pattern is observed for the BLYP-derived ZPV energies.

Table 5 CPU times (in days, hours, and minutes) required for ethylene optimization and anharmonic frequency calculations performed using the B3LYP density functional along with Dunning and segmented polarization-consistent basis sets

\begin{tabular}{|c|c|c|c|c|c|c|c|}
\hline & & \multirow{2}{*}{\multicolumn{2}{|c|}{ Number of basis functions }} & \multicolumn{4}{|l|}{ CPU time ${ }^{\mathrm{a}}$} \\
\hline & & & & \multicolumn{2}{|c|}{ Optimization } & \multicolumn{2}{|c|}{ Anharmonic frequencies } \\
\hline \multirow[t]{7}{*}{ Dunning basis sets } & $X$ & cc-pVXZ & aug-cc-pVXZ & cc-pVXZ & aug-cc-pVXZ & cc-pVXZ & aug-cc-pVXZ \\
\hline & 2 & 48 & 82 & $8 \mathrm{~m}$ & $8 \mathrm{~m}$ & $1 \mathrm{~h} 47 \mathrm{~m}$ & $3 \mathrm{~h} 35 \mathrm{~m}$ \\
\hline & 3 & 116 & 184 & $14 \mathrm{~m}$ & $16 \mathrm{~m}$ & $6 \mathrm{~h} 3 \mathrm{~m}$ & $15 \mathrm{~h} 44 \mathrm{~m}$ \\
\hline & 4 & 230 & 344 & $29 \mathrm{~m}$ & $54 \mathrm{~m}$ & $1 \mathrm{~d} 2 \mathrm{~h} 17 \mathrm{~m}$ & $3 \mathrm{~d} 13 \mathrm{~h} 26 \mathrm{~m}$ \\
\hline & 5 & 402 & 574 & $1 \mathrm{~h} 48 \mathrm{~m}$ & $6 \mathrm{~h} 59 \mathrm{~m}$ & $6 \mathrm{~d} 5 \mathrm{~h} 20 \mathrm{~m}$ & $30 \mathrm{~d} 19 \mathrm{~h} 37 \mathrm{~m}$ \\
\hline & 6 & 644 & 886 & $13 \mathrm{~h} 51 \mathrm{~m}$ & $4 \mathrm{~d} 15 \mathrm{~h} 34 \mathrm{~m}$ & $53 \mathrm{~d} 1 \mathrm{~h} 37 \mathrm{~m}$ & $226 \mathrm{~d} 19 \mathrm{~h} 14 \mathrm{~m}$ \\
\hline & & Number $\mathrm{o}$ & sis functions & Optimizatic & & Anharmonic fre & encies \\
\hline \multirow[t]{6}{*}{ Polarization-consistent basis sets } & $n$ & pcseg- $n$ & aug-pcseg- $n$ & pcseg- $n$ & aug-pcseg- $n$ & pcseg- $n$ & aug-pcseg- $n$ \\
\hline & 0 & 26 & 38 & $7 \mathrm{~m}$ & $6 \mathrm{~m}$ & $1 \mathrm{~h} 7 \mathrm{~m}$ & $1 \mathrm{~h} 27 \mathrm{~m}$ \\
\hline & 1 & 48 & 82 & $7 \mathrm{~m}$ & $9 \mathrm{~m}$ & $1 \mathrm{~h} 44 \mathrm{~m}$ & $3 \mathrm{~h} 28 \mathrm{~m}$ \\
\hline & 2 & 116 & 184 & $15 \mathrm{~m}$ & $19 \mathrm{~m}$ & $5 \mathrm{~h} 54 \mathrm{~m}$ & $15 \mathrm{~h} 49 \mathrm{~m}$ \\
\hline & 3 & 252 & 366 & $34 \mathrm{~m}$ & $1 \mathrm{~h} 2 \mathrm{~m}$ & $1 \mathrm{~d} 6 \mathrm{~g} 3 \mathrm{~h}$ & $4 \mathrm{~d} 2 \mathrm{~h} 6 \mathrm{~m}$ \\
\hline & 4 & 446 & 618 & $2 \mathrm{~h} 41 \mathrm{~m}$ & $1 \mathrm{~d} 27 \mathrm{~m}$ & $8 \mathrm{~d} 4 \mathrm{~h} 27 \mathrm{~m}$ & $42 \mathrm{~d} 15 \mathrm{~h} 45 \mathrm{~m}$ \\
\hline
\end{tabular}

${ }^{a}$ Calculations were performed using 24 processors and 50 GB of memory 


\section{CPU times required for harmonic and anharmonic calculations}

The frequency analysis presented above shows that the CBS-calculated RMS deviations in the vibrational frequencies of ethylene are nearly the same for all of the basis set families. In the last stage of our study, we probed the computational demands of each model (harmonic and anharmonic), density functional, and basis set (Table 5). It is apparent from Table 5 that employing Dunning basis sets (particularly the augmented versions) instead of the polarization-consistent ones is significantly more computationally expensive.

\section{Conclusions}

Structural parameters of ethylene were calculated using the BLYP and B3LYP density functionals. The estimated complete basis set (CBS) values were found to be the same regardless of the polarization-consistent or correlation-consistent basis set used. The $\mathrm{C}=\mathrm{C}$ and $\mathrm{C}-\mathrm{H}$ bond lengths and the $\mathrm{C}-\mathrm{C}-\mathrm{H}$ angle practically converged when basis sets with $n=2$ and $X$ $=3$ were used, and augmentation with diffuse functions did not change the CBS values. The B3LYP functional was somewhat better at predicting the structure of ethylene.

The RMS deviations of 12 CBS-estimated vibrational frequencies of ethylene from their experimentally derived values depended on the density functional used and whether a correction for anharmonicity was included. We demonstrated that the BLYP-calculated harmonic frequencies had RMS deviations that were about 3 times smaller than those of the B3LYP-calculated harmonic frequencies. Using the computationally expensive VPT2 model significantly improved the performance of B3LYP (the original RMS deviation of 77 dropped to $20 \mathrm{~cm}^{-1}$ ), but led to BLYP-calculated results that underestimated the actual value even more than before (original RMS deviation of 26 increased to $65 \mathrm{~cm}^{-1}$ ).

When the B3LYP density functional was used, the pc-4 basis set yielded near-identical anharmonic frequencies to those given by the aug-pc-4 basis set; the same was true for the basis sets with $n=2$, too. However, the results provided by aug-pc-3 were systematically overestimated (by about $20 \mathrm{~cm}^{-1}$ in the case of the $\mathrm{C}=\mathrm{C}$ stretching vibrational frequency) with respect to the results given by pc-3. This deficiency suggests that CBS frequencies cannot be reliably estimated using B3LYP/aug-pc- $n$ calculations. There was some scatter in the anharmonic frequencies and the ZPV energy values calculated using the B3LYP/cc-pVXZ and B3LYP/ aug-cc-pVXZ methods, making CBS frequency estimation unreliable. On the other hand, the newly refined aug-pcseg- $n$ series of basis sets produced regularly converging anharmonic frequencies. The Dunning basis sets yielded the same results, but required much more CPU time.
A future study aimed at checking the performance of the segmented polarization-consistent basis set family and the B3LYP density functional when using the VPT2 model to accurately reproduce the experimental vibrational spectra for a set of short polyenes of general formula $\mathrm{H}-(\mathrm{C}=\mathrm{C}-\mathrm{C}=\mathrm{C})_{n}-\mathrm{H}$ $(n=1,2,3,4)$ is needed. In addition, the use of harmonic $\mathrm{BLYP} / \mathrm{pc}-2$ calculations as a computationally less expensive alternative to VPT2 calculated at the B3LYP/pc-2 level of theory should be tested as a means of predicting trends in $\mathrm{C}=\mathrm{C}$ stretching frequencies for polyenes with $n$ values of up to 8 .

Acknowledgments T.K., A.B., and M.A.B. were supported by the Faculty of Chemistry, University of Opole (grant number 8/WCH/2013-S). Provision of the facilities and software required to perform the calculations in this work by the Supercomputing and Networking Center ACK CYFRONET AGH in Krakow (grant number MNiSW/SGI3700/ UOpolski/061/2008, PLGRID on the Zeus supercomputer) and the Supercomputing and Networking Center in Wrocław is also acknowledged.

Open Access This article is distributed under the terms of the ?Creative Commons Attribution 4.0 International License (http:// creativecommons.org/licenses/by/4.0/), which permits unrestricted use, distribution, and reproduction in any medium, provided you give appropriate credit to the original author(s) and the source, provide a link to the Creative Commons license, and indicate if changes were made.

\section{Reference}

1. Craig NC, Demaison J, Groner P, Rudolph HD, Vogt N (2015) Electron delocalization in polyenes: a semiexperimental equilibrium structure for ( $3 E)$-1,3,5-hexatriene and theoretical structures for (3Z,5Z)-, $(3 E, 5 E)-$, and (3E,5Z)-1,3,5,7-octatetraene. J Phys Chem A 119:195-204

2. Kupka T, Lin H-M, Stobinski L, Chen C-H, Liou W-J, Wrzalik R, Flisak Z (2010) Experimental and theoretical studies on corals. I. Toward understanding the origin of color in precious red corals from Raman and IR spectroscopies and DFT calculations. J Raman Spectrosc 41:651-658

3. Foresman JB, Frisch A (1996) Exploring chemistry with electronic structure methods, 2nd edn. Gaussian, Inc., Pittsburgh

4. Scott AP, Radom L (1996) Harmonic vibrational frequencies: an evaluation of Hartree-Fock, Moller-Plesset, quadratic configuration interaction, density functional theory, and semiempirical scale factors. J Phys Chem 100:16502-16513

5. Hehre WJ, Radom L, Schleyer PR, Pople JA (1986) Ab initio molecular orbital theory. Wiley, New York

6. Merrick JP, Moran D, Radom L (2007) An evaluation of harmonic vibrational frequency scale factors. J Phys Chem A 111:1168311700

7. Sinha P, Boesch SE, Gu C, Wheeler RA, Wilson AK (2004) Harmonic vibrational frequencies: scaling factors for HF, B3LYP, and MP2 methods in combination with correlation consistent basis sets. J Phys Chem A 108:9213-9217

8. Bounouar M, Scheurer C (2008) The impact of approximate VSCF schemes and curvilinear coordinates on the anharmonic vibrational frequencies of formamide and thioformamide. Chem Phys 347: 194-207

9. Bowman JM (1978) Self-consistent field energies and wavefunctions for coupled oscillators. J Chem Phys 68:608-610 
10. Bowman JM (1986) The self-consistent-field approach to polyatomic vibrations. Acc Chem Res 19:202-208

11. Gerber RB, Ratner MA (1988) Self-consistent field methods for vibrational excitation in polyatomic systems. Adv Chem Phys 70: 97-132

12. Bowman JM, Christoffel K, Tobin F (1979) Application of SCF-SI theory to vibrational motion in polyatomic molecules. J Phys Chem 83:905-920

13. Christoffel KM, Bowman JM (1982) Investigations of selfconsistent field, SCF CI and virtual state configuration interaction vibrational energies for a model three-mode system. Chem Phys Lett 85:220-224

14. Carter S, Bowman JM, Handy NC (1998) Extensions and tests of "multimode": a code to obtain accurate vibration/rotation energies of many-mode molecules. Theor Chem Acc 100:191-198

15. Christiansen O (2004) Vibrational coupled cluster theory. J Chem Phys 120:2149-2159

16. Barone V (2004) Vibrational zero-point energies and thermodynamic functions beyond the harmonic approximation. J Chem Phys 120:3059-3065

17. Begue D, Carbonniere P, Barone V, Pouchan C (2005) Performance of ab initio and DFT PCM methods in calculating vibrational spectra in solution: formaldehyde in acetonitrile as a test case. Chem Phys Lett 416:206-211

18. Barone V (2005) Anharmonic vibrational properties by a fully automated second-order perturbative approach. J Chem Phys 122: 014108

19. Barone V, Biczysko M, Bloino J, Cimino P, Penocchio E, Puzzarini C (2015) CC/DFT route toward accurate structures and spectroscopic features for observed and elusive conformers of flexible molecules: pyruvic acid as a case study. J Chem Theor Comp 11(9):4342-4363

20. Barone V, Biczysko M, Bloino J (2014) Fully anharmonic IR and Raman spectra of medium-size molecular systems: accuracy and interpretation. Phys Chem Chem Phys 16:1759-1787

21. Labanowski JK, Anzelm JW (2011) Density functional methods in chemistry. Springer, London

22. Kohn W, Sham LJ (1965) Self-consistent equations including exchange and correlation effects. Phys Rev 140:A1133-A1138

23. Dunning TH Jr (1989) Gaussian basis sets for use in correlated molecular calculations. I. The atoms boron through neon and hydrogen. J Chem Phys 90:1007-1023

24. Kendall RA, Dunning TH Jr, Harrison RJ (1992) Electron affinities of the first-row atoms revisited. Systematic basis sets and wave functions. J Chem Phys 96:6796-6806

25. Wilson A, van Mourik T, Dunning TH Jr (1996) Gaussian basis sets for use in correlated molecular calculations. VI. Sextuple zeta correlation consistent basis sets for boron through neon. J Mol Struct (Theochem) 388(1-3):339-349

26. Dunning TH Jr (2000) A road map for the calculation of molecular binding energies. J Phys Chem A 104:9062-9080

27. Jensen F (1999) The basis set convergence of the Hartree-Fock energy for H2. J Chem Phys 110(14):6601-6605

28. Jensen F (2001) Polarization consistent basis sets: principles. J Chem Phys 115(20):9113-9125

29. Jensen F (2002) Polarization consistent basis sets. II. Estimating the Kohn-Sham basis set limit. J Chem Phys 116(17):7372-7379

30. Jensen F (2003) Polarization consistent basis sets. IV. The basis set convergence of equilibrium geometries, harmonic vibrational frequencies, and intensities. J Chem Phys 118(6):2459-2463

31. Jensen F, Helgaker T (2004) Polarization consistent basis sets. V. The elements Si-Cl. J Chem Phys 121(8):3463-3470

32. Jensen $\mathrm{F}$ (2005) The effect of different density functional methods on basis set parameters. Chem Phys Lett 402(4-6):510-513
33. Jorge FE, Sagrillo PS, de Oliveira AR (2006) Gaussian basis sets of 5 zeta valence quality for correlated wave functions. Chem Phys Lett 432:558-563

34. Kupka T, Lim C (2007) Polarization-consistent vs correlationconsistent basis sets in predicting molecular and spectroscopic properties. J Phys Chem A 111:1927-1932

35. Kupka T (2008) Complete basis set B3LYP NMR calculations of $\mathrm{CDCl}_{3}$ solvent's water fine spectral details. Magn Reson Chem 46: 851-858

36. Kupka T, Stachów M, Nieradka M, Kaminsky J, Pluta T (2010) Convergence of nuclear magnetic shieldings in the Kohn-Sham limit for several small molecules. J Chem Theor Comput 6:1580 1589

37. Breidung J, Thiel W, Gauss J, Stanton JF (1999) Anharmonic force fields from analytic $\mathrm{CCSD}(\mathrm{T})$ second derivatives: $\mathrm{HOF}$ and $\mathrm{F}_{2} \mathrm{O}$. J Chem Phys 110:3687-3696

38. Bak KL, Gauss J, Jorgensen P, Olsen J, Helgaker T, Stanton JF (2001) The accurate determination of molecular equilibrium structures. J Chem Phys 114:6548-6556

39. Buczek A, Kupka T, Broda MA (2011) Extrapolation of water and formaldehyde harmonic and anharmonic frequencies to the B3LYP/ CBS limit using polarization consistent basis sets. J Mol Model 17: 2029-2040

40. Buczek A, Kupka T, Broda MA (2011) Estimation of formamide harmonic and anharmonic modes in the Kohn-Sham limit using the polarization consistent basis sets. J Mol Model 17:2265-2274

41. Buczek A, Kupka T, Sauer SPA, Broda MA (2012) Estimating the carbonyl anharmonic vibrational frequency from affordable harmonic frequency calculations. J Mol Model 18:2471-2478

42. Broda MA, Buczek A, Kupka T, Kaminsky J (2012) Anharmonic frequencies of solvated molecules in the complete basis set limit. Vibr Spectrosc 63:432-439

43. Tomasi J, Mennucci B, Cammi R (2005) Quantum mechanical continuum solvation models. Chem Rev 105:2999-3093

44. Martin JML, Taylor PR (1996) The geometry, vibrational frequencies, and total atomization energy of ethylene. A calibration study. Chem Phys Lett 248:336-344

45. Martin J, Lee T, Taylor P, Francois J-P (1995) The anharmonic force field of ethylene, $\mathrm{C}_{2} \mathrm{H}_{4}$, by means of accurate ab initio calculations. J Chem Phys 103:2589-2602

46. Feller D, Peterson KA, Dixon DA (2008) A survey of factors contributing to accurate prediction of atomization energies and molecular structures. J Chem Phys 129:204105

47. Craig NC, Groner P, McKean DC (2006) Equilibrium structures for butadiene and ethylene: compelling evidence for $\pi$-electron delocalization in butadiene. J Phys Chem A 110:7461-7469

48. Nozirov F, Kupka T, Stachów M (2014) Theoretical prediction of nuclear magnetic shieldings and indirect spin-spin coupling constants in 1,1-, cis, and trans-1,2-difluoroethylenes. J Chem Phys 140:144303

49. Becke AD (1993) Density-functional thermochemistry. III. The role of exact exchange. J Chem Phys 98(7):5648-5652

50. Lee C, Yang W, Parr RG (1988) Development of the Colle-Salvetti correlation-energy formula into a functional of the electron density. Phys Rev B 37(2):785-789

51. Miehlich B, Savin A, Stoll H, Preuss H (1989) Results obtained with the correlation-energy density functionals of Becke and Lee, Yang and Parr. Chem Phys Lett 157:200-206

52. Becke AD (1988) Density-functional exchange-energy approximation with correct asymptotic behavior. Phys Rev A 38:3098-3100

53. Krishnan R, Binkley JS, Seeger R, Pople JA (1980) Self-consistent molecular orbital methods. XX. A basis set for correlated wave functions. J Chem Phys 72:650-654

54. Frisch MJ, Pople JA, Binkley JS (1984) Self-consistent molecular orbital methods. 25. Supplementary functions for Gaussian basis sets. J Chem Phys 80:32653269 
55. Jensen F (2014) Unifying general and segmented contracted basis sets. Segmented polarization consistent basis sets. J Chem Theory Comput 10:1074-1085

56. Frisch MJT, Trucks GW, Schlegel HB et al (2009) Gaussian 09, revision D.01. Gaussian, Inc., Wallingford

57. Schuchardt KL, Didier BT, Elsethagen T, Sun L, Gurumoorthi V, Chase J, Li J, Windus TL (2007) Basis Set Exchange: a community database for computational sciences. J Chem Inf Model 47:10451052

58. EMSL (2015) Basis Set Exchange. Environmental Molecular Sciences Laboratory, Richland. https://bse.pnl.gov/bse/portal

59. Feller D (1996) The role of databases in support of computational chemistry calculations. J Comp Chem 17:1571-1586

60. Frisch MJT, Trucks GW, Schlegel HB et al (2009) Gaussian 09, revision A.02. Gaussian, Inc., Wallingford

61. Duncan JL, Hamilton E (1981) An improved general harmonic force field for ethylene. J Mol Struct 76:65-80

62. Van Lerberghe D, Wright IJ, Duncan JL (1972) High-resolution infrared spectrum and rotational constants of ethylene-H4. J Mol Spectrosc 42:251-273

63. Martin JML (1998) Encyclopedia of computational chemistry. vol 5. Wiley New York

64. Feller D (1992) Application of systematic sequences of wave functions to the water dimer. J Chem Phys 96(8):6104-6114

65. Feller D (1993) The use of systematic sequences of wave functions for estimating the complete basis set, full configuration interaction limit in water. J Chem Phys 98(9):7059-7071

66. Helgaker T, Klopper W, Koch H, Noga J (1997) Basis-set convergence of correlated calculations on water. J Chem Phys 106(23): 9639-9646

67. Puzzarini C (2009) Extrapolation to the complete basis set limit of the structural parameters. Comparison of different approaches. J Phys Chem A 113:14530-14535
68. Puzzarini C, Barone V (2010) Benchmark calculations for molecules in the gas phase: state-of-the-art coupled-cluster computations. Int J Quant Chem 110:637-655

69. Kupka T, Stachów M, Chełmecka E, Pasterny K, Stobiński L (2012) DFT calculations of structures, ${ }^{13} \mathrm{C}$ NMR chemical shifts and Raman RBM mode of simple models of ultra small diameter (4.0) zigzag hydroxylated single wall carbon nanotubes. Synth Metals 162:573-583

70. Kupka T, Ruscic B, Botto RE (2002) Toward Hartree-Fock- and density functional complete basis-set predicted NMR parameters. J Phys Chem A 106:10396-10407

71. Karampelas S, Fritsch E, Mevellec J-Y, Gauthier J-P, Sklavounos S, Soldatos T (2007) Determination by Raman scattering of the nature of pigments in cultured freshwater pearls from the mollusk Hyriopsis cumingi. J Raman Spectrosc 38:217-230

72. Shimanouchi T (1972) Tables of molecular vibrational frequencies Consolidated. Vol 1, Washington, DC. National Bureau of Standards

73. Nozirov F, Kupka T, Stachów M (2014) Theoretical prediction of structural, vibrational and NMR properties of plastic optical fiber (POF) material precursors. Cis- and trans-perhydro- and perfluoro2-methylene-4,5-dimethyl-1,3-dioxolanes. J Mol Graphic Model 52:36-45

74. Matisz G, Kelterer, AM, Fabian, WMF, Kunsági-Máté, S (2011) Coordination of methanol clusters to benzene: a computational study. J Phys Chem A 115(38):10556-10564

75. Kupka T, Ruscic B, Botto RE (2003) Hartree-Fock and density functional complete basis-set (CBS) predicted nuclear shielding anisotropy and shielding tensor components. Solid State Nucl Magn Reson 2:143-167

76. NIST (2015) CCCBDB: CCSD(T)/aQZ harmonic results. http:// cccbdb.nist.gov/energy $3 \mathrm{x}$.asp? method $=63 \&$ basis $=26 \&$ charge $=0$. Accessed 15 Nov 2015 